\title{
Exoplanet Searches in the Habitable Zone with Gravitational Microlensing
}

\author{
Alexander F. Zakharov ${ }^{1,2} \dagger$, Gabriele Ingrosso In, $^{3,4}$ \\ Francesco De Paolis ${ }^{3,4}$, Achille A. Nucita ${ }^{3,4}$, Francesco Strafella ${ }^{3}$, \\ Sebastiano Calchi Novati ${ }^{5,6}$, Philippe Jetzer ${ }^{7}$ \\ ${ }^{1}$ Institute of Theoretical and Experimental Physics, Moscow, 117259, Russia \\ ${ }^{2}$ Bogoliubov Laboratory of Theoretical Physics, JINR, Dubna, Russia \\ ${ }^{3}$ Dipartimento di Matematica e Fisica "Ennio De Giorgi", Università del Salento, CP 193, \\ I-73100 Lecce, Italy \\ ${ }^{4}$ INFN Sezione di Lecce, CP 193, I-73100 Lecce, Italy \\ ${ }^{5}$ Dipartimento di Fisica "E.R. Caianiello", Università di Salerno, I-84081 Baronissi (SA), Italy \\ ${ }^{6}$ Istituto Internazionale per gli Alti Studi Scientifici (IIASS), Vietri Sul Mare (SA), Italy \\ ${ }^{7}$ Institute for Theoretical Physics, University of Zürich, CH-8057, Zürich, Switzerland
}

\begin{abstract}
There are different methods for finding exoplanets such as radial spectral shifts, astrometrical measurements, transits, timing, etc. Gravitational microlensing (including pixellensing) is among the most promising techniques with the potential of detecting Earth-like planets at distances about a few astronomical units from their host stars. Here we emphasize the importance of polarization measurements which can help to resolve degeneracies in theoretical models. In particular, the polarization angle could give additional information about the relative position of the lens with respect to the source.
\end{abstract}

Keywords. Gravitational lensing, Microlensing, Extrasolar planets

Before the discovery of the first exoplanet, Mao \& Paczynski (1991) had shown how efficient gravitational microlensing is in search for extrasolar planets, including the lowmass ones, even at relatively large distances from their host stars. Later on, observations and simulations gave the opportunity to confirm the robustness of Mao \& Paczynski (1991) conclusions. Exoplanets near the snow-line may be also detected with this technique as it was shown, for instance, in Fig. 8 in Mao (2012). Moreover, in contrast with conventional methods, such as transits and Doppler shift measurements, gravitational microlensing gives a chance to find exoplanets not only in the Milky Way (Beaulieu et al. 2006; Dominik 2010; Wright \& Gaudi 2013; Gaudi 2012; Mao 2012), but also in nearby galaxies, such as the Andromeda galaxy (Ingrosso et al. 2009, 2011). Pixel-lensing towards M31 provides an efficient tool to search for exoplanets and indeed an exoplanet might have been already discovered in the PA-N2-99 event (An et al. 2004; Ingrosso et al. 2009). Moreover, since source stars for pixel-lensing towards M31 are basically red giants (and therefore, their typical diameters are comparable to Einstein diameters and the caustic sizes) one has to take into account the source finiteness effect (Pejcha \& Heyrovský 2009). In the case of relatively small size sources, the probability to have features due to binary lens (or planet around star) in the light curves is also small since it is proportional to the caustic area. Giant star sources have large angular sizes and relatively higher probability to touch caustics (See for instance Ingrosso et al. 2009, for details). 


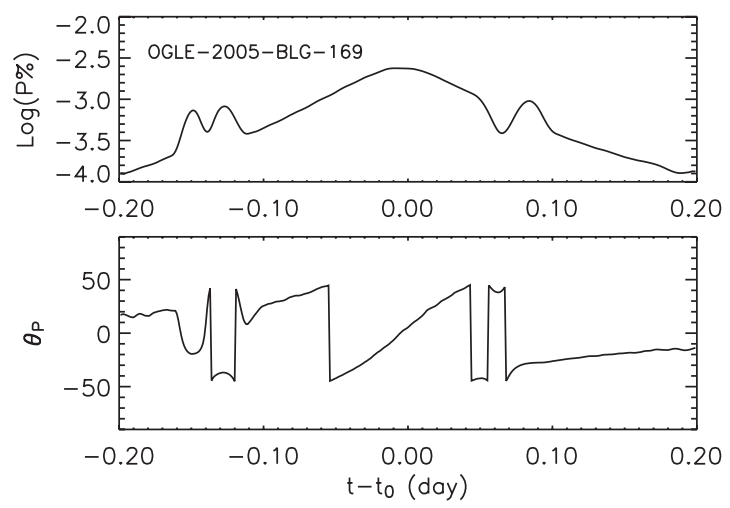

Figure 1. Polarization curve (top panel) and polarization angle (bottom panel) for the OGLE-2005-BLG-169 event.

For extended sources, the importance of polarization measurements was pointed out by Bogdanov, Cherepashchuk \& Sazhin (1996) for point-like lens and by Agol (1996) for binary lens (see also Ignace, Bjorkman \& Bryce 2006). For point-like lens polarization could reach $0.1 \%$ while for binary lens it could reach a few percent since the magnification gradient is much greater near caustics. It has been shown that polarization measurements could resolve degeneracies in theoretical models of microlensing events (Agol 1996). Calculations of polarization curves for microlensing events with features in the light curves induced by the presence of an exoplanet and observed towards the Galactic bulge have been done (Ingrosso et al. 2012). Here we emphasize that measurements of the polarization angle could give additional information about the gravitational microlensing model. For instance, for a point-like lens the direction for the maximal polarization (which is perpendicular to the line connecting star and lens) may allow to infer the direction of lens proper motion, does allowing to eventually pinpoint the lens in following observations. Even in the case of binary lens, the orientation of polarization vector corresponds to the orientation of the fold caustic (or more correctly to the tangent vector to the fold caustic at the intersection point with the path of source), provided the source size is small enough.

In Fig. 1, the polarization curve and the polarization angle are shown for the OGLE2005-BLG-169 event, where a binary system formed by a main sequence star with mass $M_{\odot} \sim 0.5 M_{\odot}$ and a Neptune-like exoplanet with mass about $13 M_{\oplus}$ is expected from the light curve analysis (Gould et al. 2006). The event parameters are $t_{E}=42.27$ days, $u_{0}=1.24 \times 10^{-3}, b=1.0198, q=8.6 \times 10^{-5}, \alpha=117.0 \mathrm{deg}, \rho_{*}=4.4 \times 10^{-4}$, where $t_{E}, u_{0}, b, q, \alpha, \rho_{*}$ are the Einstein time, the impact parameter, the projected distance of the exoplanet to the host star, the binary component mass ratio, the angle formed by the source trajectory and the separation vector between the lenses, and the source star size, respectively (all distances are given in $R_{E}$ units). The effect of the source transiting the caustic (see Gould et al. (2006)) is clearly visible both in the polarization curve (see top panel in Fig. 1) and in the flip of the polarization angle (see bottom panel). We would like to stress that the high peak magnification $(A \simeq 800)$ of the OGLE-2005BLG-169 event leading to I-magnitude of the source about 13 mag at the maximum gives the opportunity to measure the polarization signal for such kind of events by using present available facilities. In this case, polarization measurements might give additional information about the caustic structure, thus potentially allowing to distinguish among different models of exoplanetary systems. 

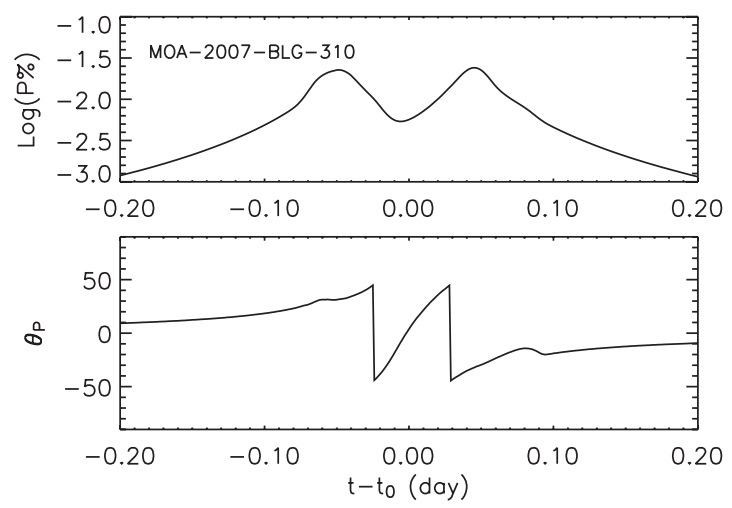

Figure 2. Polarization curve (top panel) and the polarization angle (bottom panel) for the MOA-2008-BLG-310Lb.

The polarization curve and the polarization angle for the MOA-2008-BLG-310Lb event is shown in Fig. 2. For this event it was expected the existence of a sub-Saturn exoplanet with mass $m=74 \pm 17 M_{\oplus}$ (Janczak et al. 2010). The event parameters are $t_{E}=$ 11.14 days, $u_{0}=3 . \times 10^{-3}, b=1.085, q=3.31 \times 10^{-4}, \alpha=69.33 \mathrm{deg}, \rho_{*}=4.93 \times 10^{-3}$. In particular, the event is characterized by large finite source effect since $\rho_{*} / u_{0}>1$, leading to polarization features similar to those of single lens events. Nevertheless, in this case we can see the variability in the polarization signal that arises when the source touches the first fold caustic at $t_{1} \simeq t_{0}-0.07$ days, the source enters the primary lens at $t_{2} \simeq t_{0}-t_{E} \sqrt{\rho_{*}^{2}-u_{0}^{2}}$ days, the source exits the primary lens at $t_{3} \simeq t_{0}+t_{E} \sqrt{\rho_{*}^{2}-u_{0}^{2}}$ days and touches the second fold caustic $t_{4} \simeq t_{0}+0.09$ days (see also Fig. 4 in Janczak et al. (2010)).

Recently, Gould et al. (2013) found that a variable giant star source mimics exoplanetary signatures in the MOA-2010-BLG-523S event. In this respect, we emphasize that polarization measurements may be helpful in distinguishing exoplanetary features from other effects in the light curves.

\section{References}

Agol, E., 1996, MNRAS, 279, 571

An J. H. et al., 2004, ApJ, 601, 845

Beaulieu, J.-P. et al., 2006, Nature, 644, L37

Bogdanov, M. B., Cherepashchuk, A. M., \& Sazhin, M. V. 1996, Ap\&SS, 235, 219

Dominik, M., 2010, Gen. Rel. Grav., 42, 2075

Gaudi, B. S., 2012, Annual Rev. Astron. \&3 Astrophys., 50, 411

Gould, A. et al., 2006, ApJ, 644, L37

Gould, A. et al., 2013, ApJ, 763, article id. 141

Ignace, R., Bjorkman, E., \& Bryce, H. M., 2006, MNRAS, 366, 92

Ingrosso, G. et al., 2009, MNRAS, 399, 219

Ingrosso, G. et al., 2011, Gen. Rel. Grav., 43, 1047

Ingrosso, G. et al., 2012, MNRAS, 426, 1496

Janczak, J. et al., 2010, ApJ, 711, 731

Mao, S. 2012, Res. Astron. Astrophys. 12, 947

Mao, S. \& Paczynski, B., 1991, ApJ, 374, L37

Pejcha, O. \& Heyrovský D., 2009, ApJ, 690, 1772

Wright, J. T. \& Gaudi, B. S., 2013, in Planets, Stars, and Stellar Systems, eds. T. D. Oswalt, L. M. French, P. Kalas (Springer, Dordrecht), 489 